\title{
Spitzer observations of molecules and dust in evolved stars in nearby galaxies
}

\section{Mikako Matsuura}

Department of Physics and Astronomy, University College London, Gower Street, London

WC1E 6BT, United Kingdom; email: mikako@star.ucl.ac.uk

\section{Introduction}

Molecules and dust are formed in and around the asymptotic giant branch (AGB) stars and supernovae (SNe), and are ejected into the interstellar medium (ISM) through the stellar wind. The dust and gas contain elements newly synthesised in stars, thus, dying stars play an important role in the chemical enrichment of the ISM of galaxies. However, quantitative analysis of molecules and dust in these stars had been difficult beyond our Galaxy. The high sensitivity instruments on-board the Spitzer Space Telescope (SST; Werner et al. 2004) have enabled us to study dust and molecules in these stars in nearby galaxies. Nearby galaxies have a wide range in metallicity, thus the impact of the metallicity on dust and gas production can be studied. This study will be useful for chemical evolution of galaxies from low to high metallicity.

\section{Observations and results}

We present $5-35 \mu \mathrm{m}$ spectra, obtained by the infrared spectrometer (IRS) on-board the $S S T$. Targets are mainly asymptotic giant branch (AGB) stars located in five nearby galaxies within $200 \mathrm{kpc}$. The main objectives are: 1) to exploit the dust property, molecular abundance, and mass loss of AGB stars for a wide range of metallicities of the host galaxies, and 2) to investigate the contribution of mass loss from AGB stars on the galactic chemical evolution. The metallicities of these galaxies range from half of the solar metallicity (Large Magellanic Cloud) to $5 \%$ of the solar metallicity (Sculptor dwarf spheroidal galaxy). We found the following results.

- Carbon-rich AGB stars have abundant $\mathrm{C}_{2} \mathrm{H}_{2}$ molecules in low metallicity galaxies (Matsuura et al. 2005, 2006, 2007; Sloan et al. 2009). At low metallicity, as the initial oxygen abundance is low, after carbon elements are synthesised in stars, it leads to a high carbon-to-oxygen $(\mathrm{C} / \mathrm{O})$ abundance ratio in carbon-rich AGB stars. A similar effect is found for amorphous carbon (Groenewegen et al. 2007).

- The $\mathrm{SiC}$ dust fraction is lower at lower metallicity, but this fraction suddenly increases at the end of the AGB phase (Gruendl et al. 2008). The dust condensation sequence ( $\mathrm{SiC}$, and amorphous carbon/graphite) changes at low metallicity.

- We found that the gas mass ejected from all AGB stars and SNe almost the same in the LMC (Matsuura et al. 2009). AGB stars are one of the major dust sources in the LMC. AGB dust at low metallicity tends to be more carbonaceous than oxidized.

\section{References}

Groenewegen M. A. T., et al., 2007, MNRAS 376, 313

Gruendl R. A. et al., 2008, ApJ 688, L9

Matsuura M., et al., 2005, A\&\&A 434, 691

Matsuura M., et al., 2006, MNRAS 371, 415

Matsuura M., et al., 2007, MNRAS 382, 1889

Matsuura M., et al., 2009, MNRAS 396, 918

Sloan G. C., et al., 2009, Science 323, 353

Werner M. W., et al., 2004, ApJS 154, 1 